Published in final edited form as:

Alzheimers Dement. 2008 March ; 4(2): 89-93.

\title{
NEW DIRECTIONS FOR FRONTOTEMPORAL DEMENTIA DRUG DISCOVERY
}

\author{
John Q. Trojanowski ${ }^{1^{*}}$, Karen Duff ${ }^{2}$, Howard Fillit ${ }^{3}$, Walter Koroshetz ${ }^{4}$, Jeff Kuret ${ }^{5}$, Diane \\ Murphy ${ }^{4}$, and Larry Refolo 4 \\ 1Institute on Aging, Center for Neurodegenerative Disease Research, and Department of Pathology and \\ Laboratory, University of Pennsylvania School of Medicine, Philadelphia, PA \\ 2Department of Pathology, Columbia University College of Physicians and Surgeons, New York, NY \\ 3Institute for the Study of Aging, New York NY \\ 4The National Institute of Neurological Disease and Stroke of the National Institutes of Health, Bethesda, \\ $M D$
}

5Ohio State University, Columbus, $\mathrm{OH}$

\begin{abstract}
This report summarizes the recommendations of the Frontotemporal Dementia (FTD) Working Group on FTD Drug Discovery that was part of an international FTD Workshop held in January 18th and 19th, 2007 in Miami, Florida. The workshop was sponsored by the National Institute of Neurological Disorders and Stroke (NINDS) and the National Institute on Aging (NIA) of the National Institutes of Health (NIH) and the Association for Frontotemporal Dementia (AFTD) with the express purpose of defining opportunities to improve the diagnosis and treatment of patients affected by a neurodegenerative disorder classified as one of the many variants of FTD. Recognition that almost all forms of FTD are due to TDP-43 proteinopathies and tauopathies creates new opportunities for FTD drug discovery targeting pathways of TDP-43 and tau mediated neurodegeneration as reviewed here.
\end{abstract}

\section{Background On FTD And Mechanisms of Neurodegeneration1}

Most neurodegenerative disorders are characterized by abnormal protein aggregates in neurons and glia of the central nervous system (CNS) [17]. The identification of disease-specific abnormal protein inclusions has catalyzed efforts to elucidate mechanisms of pathogenesis and molecular classification of neurodegenerative diseases as exemplified by the two most common of these disorders, Alzheimer's disease (AD) and Parkinson's disease (PD), but also less common disorders such as Frontotemporal dementias (FTDs), which account for $20 \%$ of presenile dementia cases $[3,10]$. Although FTDs are clinically, genetically, and neuropathologically heterogeneous, $>95 \%$ of cases are TDP43 proteinopathies or tauopathies,

\footnotetext{
*Corresponding author, Institute on Aging \& Center for Neurodegenerative Disease Research, Department of Pathology and Laboratory Medicine, University of Pennsylvania School of Medicine, HUP, Maloney 3rd Floor, 36th and Spruce Streets, Philadelphia, PA 19104-4283 USA, Tel: 215-662-6399; Fax: 215-349-5909, E-mail: trojanow @ mail.med.upenn.edu.

Publisher's Disclaimer: This is a PDF file of an unedited manuscript that has been accepted for publication. As a service to our customers we are providing this early version of the manuscript. The manuscript will undergo copyediting, typesetting, and review of the resulting proof before it is published in its final citable form. Please note that during the production process errors may be discovered which could affect the content, and all legal disclaimers that apply to the journal pertain.

${ }^{1}$ Due to rapid advances in understanding the molecular and genetic basis of FTDs, reference is made to recent reviews of research in this field wherein additional citations to primary reports are found, and selected primary paper related to FTD drug discovery are emphasized in the literature cited here.
} 
as summarized briefly here, thereby providing potential molecular targets for FTD drug discovery efforts.

Frontotemporal lobar degeneration (FTLD) with ubiquitin-positive, tau-negative inclusions (FTLD-U) was thought until recently to be the underlying pathology in about half of clinical FTDs including forms of FTD that present with motor neuron disease (MND), which is referred to as FTD-MND or FTLD-U with MND [3,10]. However, as recently reviewed elsewhere [3, 10], TAR-DNA-binding protein 43 (TDP-43), a nuclear protein implicated in exon skipping and transcription regulation, was recently identified as the major pathological protein in inclusions of sporadic and familial FTLD-U with and without MND as well as in sporadic forms of amyotrophic lateral sclerosis (ALS) [13]. Thus, TDP-43 proteinopathy may replace the other earlier terms mentioned above in the same way that tauopathies refers to another major group of FTDs characterized by tau pathologies (see below). Briefly, pathological TDP-43 in these disorders is abnormally phosphorylated, ubiquitinated and cleaved to generate C-terminal fragments, and is recovered only from affected CNS regions including hippocampus, neocortex, and spinal cord $[3,10]$. Thus, this pathology defines a novel class of neurodegenerative diseases that can be referred to as TDP-43 proteinopathies.

In most recenet studies, $\sim 50 \%$ of FTD patients will be shown at postmortem exam to have FTLD-U and FTLD-U with MND, also called FTLD-MND. The majority of cases of FTLD$\mathrm{U}$ have variable densities of ubiquitinpositive neuronal cytoplasmic inclusions (NCIs) and dystrophic neurites (DNs) in frontal and temporal neocortex and, to a lesser degree, in the parietal cortex while the occipital lobe is usually spared [3,10]. Ubiquitin-immunoreactive NCIs are typically found in the granule neurons of the dentate fascia of most cases of FTLDU and FTLD-U with MND. Neuronal intranuclear inclusions (NIIs) have been reported most commonly in familial cases of FTLD-U, especially those associated with progranulin gene $(P G R N)$ mutations, but are largely albeit not completely absent from sporadic cases.

Molecular genetic heterogeneity is a striking feature of FTLD-U. Recently, the molecular genetic causes of familial FTLD-U linked to chromosome 17 were discovered to be mutations in the $P G R N$ gene [3]. Although ubiquitin- and TDP-43-positive neuronal and glial inclusions have been found in the inclusions of familial FTLD-U with $P G R N$ mutations, progranulin proteins, as investigated by immunohistochemistry, are not components of the ubiquitin positive inclusions implying that another ubiquitinated disease protein must aggregate in these lesions, and this disease protein now is known to be TDP-43 [3,10]. Abnormal hyperphosphorylated and truncated TDP-43 has been detected biochemically in both affected gray and white matter suggesting that both glial and neuronal pathology may contribute to the pathogenesis of FTLD-U caused by PRGN mutations [10].

Inclusion body myopathy associated with Paget's disease of bone and FTD (IBMPFD) is a rare autosomal dominant disorder with mutations in the valosin-containing protein $(V C P)$ [10]. VCP, a member of the AAA-ATPase gene super family (ATPase Associated with diverse cellular Activities) has multiple cellular functions including acting as a molecular chaperone in endoplasmic reticulum-associated protein degradation (ERAD), stress response, programmed cell death, and interactions with the ubiquitin-proteasome system. In FTLD-U with VCP mutations, TDP-43 co-localizes with ubiquitin pathology both in NII and DN. Similar to FTLD-U, phosphorylated TDP-43 was detected only in the insoluble brain extracts from affected regions indicating that the $V C P$ gene mutations cause a dominant negative loss of function or alteration of VCP function leading to impaired degradation of TDP-43.

Mutations in the charged multivesicular body protein $2 B(C H M P 2 B)$ gene were recently identified as the cause of FTD linked to chromosome 3 in a large Danish pedigree. Human CHMP2B is a protein of 213 amino acids with a predicted coil-coil domain and is a component 
of the endosomal secretory complex required for transport III (ESCRITIII). Neuropathology reveals ubiquitin-positive granular NCIs in frontal neocortex and hippocampus, but no ubiquitin or TDP-43 positive DN or NII [3,10]. Recently, a new genetic locus on chromosome $9 \mathrm{p}$ for familial FTD-MND has been described. In one family, candidate gene sequencing revealed the presence of a putative disease segregating stop mutation $(\mathrm{Q} 342 \mathrm{X})$ in the intraflagellar transport protein 74 (IFT74) gene [3,10]. IFT74 is a 600 amino acid coiled-coil domain-containing protein that localizes to the intracellular vesicle compartment and is a component of the intraflagellar transport system responsible for vesicular transport of material synthesized within the cell body into and along dendrites and axons. Neuropathology of FTLDU with IFT74 mutation reveals all the stigmata of FTLD-U (ubiquitinated NCI, DN, and NII), but loss of motor neurons was not a feature of the case reported. Emerging data indicate that TDP-43 is a common pathologic substrate in a spectrum of FTDs including sporadic FTLD$\mathrm{U}$ with and without MND, familial FTLD-U with $P G R N$ and $V C P$ mutations as well as sporadic and familial forms of ALS. Thus, despite the clinical, genetic, and neuropathologic heterogeneity of FTLD, TDP-43 is the disease protein underlying a large subset of these disorders thereby implicating TDP-43 in novel and unifying mechanisms of FTLDs $[3,10]$.

Most insights into tauopathies have come from studies of AD, but prominent tau abnormalities are recognized as the defining neuropathology in close to half of clinically diagnosed FTDs cases, and these are referred to as tauopathies due to accumulations of insoluble hyperphosphorylated tau proteins in neurons and/or glial cells $[4,8,12,16,17]$. For example, besides atrophy of the frontal and temporal lobes with superficial spongiosis, Pick's disease (PiD) is characterized by large numbers of tau-positive spherical cytoplasmic inclusions called Pick bodies that are encountered in particular in the hippocampus. The main neuropathological finding in progressive supranuclear palsy (PSP) is a high density of tau-positive globose-type neurofibrillary tangles (NFTs) and neuropil threads in several subcortical structures including subthalamic nucleus, pallidum, substantia nigra or pons, and, tau positive tufted astrocyte are characteristic of this disorder, as is degeneration in the cerebellar dentate nucleus. In addition to neuronal loss in focal cortical regions, striatum and substantia nigra, corticobasal degeneration (CBD) is characterized by achromatic balloon-shaped neurons (referred to as "ballooned neurons") and by diffuse or granular cytoplasmic tau-immunoreactivity (pretangles) or small NFTs. Importantly, in CBD profound glial pathology including astrocytic plaques in cortex and thread-like lesions as well as glial tau inclusions in gray and white matter. The distinct neuropathological feature of argyrophilic grain disease $(\mathrm{AgD})$ includes the presence of abundant spindle-shaped argyrophilic grains in neuronal processes and coiled bodies. Coiled bodies occur in PSP, CBD and AGD. NFT predominant dementia (NFTPD) is characterized by abundant allocortical tau positive NFTs with no or few isocortical tau lesions, absence of neuritic plaques, and scarce amyloid- $\beta(\mathrm{A} \beta)$ deposits. Hence, these sporadic disorders are grouped together as FTD tauopathies.

After studies showed linkage of chromosome 17q21-22 to disease in several families with hereditary FTDs, additional families with a remarkable variety of clinical and pathologic phenotypes also were reported to show linkage to this region of chromosome 17 that harbors the microtubule (MT) associated protein tau (MAPT) gene [17]. Since some of these familial disorders also showed evidence of parkinsonism, they have been collectively referred to as FTD with parkinsonism linked to chromosome 17 (FTDP-17), and the major neuropathological features of FTDP-17 are neuronal and glial fibrillary tau lesions with little or no A $\beta$ pathology or other diagnostic brain abnormalities. The suspicion that FTDP-17 syndromes might be caused by autosomal dominant MAPT gene mutations was substantiated by a series of remarkable discoveries beginning in 1998 showing there now are >35 mutations in the MAPT gene that are pathogenic for these disorders. Thus, these findings provided unequivocal proof that tau abnormalities are sufficient to cause neurodegenerative diseases. 
The microtubule (MT) binding protein tau is the major constituent of NFTs, a hallmark of AD and other neurodegenerative diseases including sporadic tauopathies and autosomal dominantly inherited FTDP-17 [4,8.12,16,17]. Thus, tau pathology is a critical underlying abnormality that links all of these disorders to a shared mechanism of neurodegeneration. For example, when tau becomes hyperphosphorylated it forms paired-helical fragments which go on to form protein aggregates, or NFTs, leaving less tau available to stabilize MTs and when MTs, which are essential for intracellular transport, become compromised, neurodegeneration may follow. Several types of interventions, some impacting tau and some directly targeting the MTs could become disease modifying therapies for AD and FTD tauopathies as review below.

\section{Plausible Targets For FTD Drug Discovery Based On Mechanisms Of Disease}

The two dominant underlying pathologies of FTDs are TPD43 proteinopathies and tauopathies. Since the former is a newly discovered FTD disease protein, it is difficult to formulate strategies for drug discovery specifically targeting TDP43 pathology. However, this new FTD research field is advancing rapidly and it is plausible that FTDs caused by TDP43 proteinopathy will benefit from neuroprotective therapies in the absence of clear understanding of mechanisms of degeneration in these forms of FTD. On the other hand, considerable insight into mechanisms of neurodegenerative tauopathies suggest a number of plausible targets for tau focused drug discovery. Hence, tau focused targets for FTD drug discovery are summarized first below, followed by a discussion of neuroprotective therapeutic strategies that could benefit both tauopathies and TDP43 proteinopathies.

\section{Tau Kinases}

By inhibiting phosphorylation of tau, the formation of NFTs might be retarded and MTs could be stabilized by increasing levels of non-phosphorylated tau. Based on this view, there are several candidate kinases that may make good drug targets:

\section{Glycogen Synthase Kinase (GSK3) 3-beta (GSK3beta)}

There is considerable evidence showing that GSK3 phosphorylates tau and hence could be target for inhibition to prevent pathological tau phosphorylation in AD and FTD tauopathies $[2,4,7,14]$. GSK3beta may also cooperate with other, non-tau proteins, to contribute to neuronal disruption. There is also evidence that GSK3beta might be activated in an A $\beta$-dependent manner and that GSK3beta can promote production of beta-amyloid. Thus, this kinase may have effects on neuritic dystrophy that are independent of tau. Potent small molecule inhibitors of GSKbeta have been developed and some appear to be highly specific for GSK3 while not appreciably affecting other kinases $[2,4,7,14,16,17]$. These molecules inhibit tau phosphorylation in vitro and in vivo and they prevent tau aggregates in transgenic (Tg) mice expressing aggregate prone tau mutants. They also prevent formation of tau filaments in cultured neuroblastoma cells and they prevent tau phosphorylation in mouse models of high GSK3beta activity. While studies of small molecule inhibitors of GSK3 are a priority for AD and FTD tauopathies, there are several reasons to consider a trial of the GSK3 inhibitors lithium chloride and valproic acid for FTDs. Indeed, since they are to be tested in AD patients in the NIA funded AD Cooperative Study (ADCS), an FTD clinical trial of one or both of these compounds could benefit from synergies with similar studies conducted in the ADCS.

\section{Cyclin-dependent Kinase 5 (CKD5)}

CDK5 phosphorylates tau at several sites that have been linked to disease and it may be an ideal target for drug discovery because it is kept in its active form not by phosphorylation, but by the binding of a protein partner called p25 $[1,4,16,17]$. Screens for small molecules that 
inhibit this kinase in an ATP site-independent manner have turned up three classes of molecules. One of these comprised inhibitors that are non-competitive with respect to ATP, but competitive with respect to tau. These inhibitors may be highly specific and an ideal class of compounds for further study, but other inhibitors of tau phosphorylation may be discovered that work through other mechanisms [11].

\section{Heat Shock Proteins (HSPs) and the Ubiquitin Proteasome System}

The ubiquitin ligase carboxy terminus of Hsp70 interacting protein (CHIP) can polyubiquitinate tau and may play a crucial role in preventing accumulation of phospho-tau and NFTs [5,6]. CHIP-negative mice accumulate phospho-tau in many areas of the brain, including the cortex and hippocampus. However, even though phospho-tau accumulates in CHIP-negative animals, NFT pathology is reduced, suggesting that ubiquitination may play a role in tangle maturation. This opens up a potential strategy of preventing polyubiquitination as means to attenuate NFTs. One potential strategy is to induce cells to produce more HSPs. Inhibitors of Hsp90, for example, might causes multi-chaperone complexes to dissociate thereby releasing heat shock factor 1 (HSF-1). This transcription factor stimulates de-novo synthesis of heat shock proteins such as Hsp70, which can modulate the fate of ubiquitinated tau. In fact, Hsp90 inhibitors do lead to increased $\mathrm{Hsp} 70$ and a reduction in total phospho-tau protein in cells, suggesting they may also work in vivo.

\section{Inhibitors of Fibrillization}

The formation of the tau filaments in NFTs occurs in two steps, nucleation and extension so small molecules may be able to inhibit either step in this fibrillization process although the kinetics is often complex [9,15-17]. For example, the rate limiting step for tau fibrillization is nucleation, and this can be exponentially increased by adding some kind of nucleation inducer, such as an anionic surfactant. Small antagonists of fibrillization can work by increasing the critical concentration needed for extension of the filament into polymers. Important caveats for consideration are that such inhibition may be overcome by phosphorylation of tau or by increased tau concentration. The inhibitor/tau relationship may also be complex, with inhibitor dimerization/multimerization explaining the inhibitor dose response curves, which show reduced efficacy at higher inhibitor concentrations.

\section{MT Stabilizing Agents}

Stabilizing agents, such as paclitaxel, might help to maintain MT integrity by mimicking tau to keep tubulin in the polymerized state [19]. Numerous MT stabilizing agents have been discovered but they are often isolated from natural sources and are extremely rare. Studies are underway to screen derivatives of known MT stabilizing agents to find compounds that have ideal pharmacokinetic properties. Some of these compounds work synergistically with paclitaxel, indicating that combinations of compounds may be an avenue worth exploring.

\section{Neuroprotective Therapies for FTDs}

Since AD and FTD tauopathies are associated with a variable inflammatory response that may be induced by the underlying pathology regardless of the disease protein involved, it is plausible that immunosuppressant drugs could have beneficial effects in tauopathies and perhaps also in FTD TDP43 proteinopathies. Indeed, recent studies in tau Tg mice showed that treating these mice with the immunosupressant FK506 before disease onset had a dramatic effect on the tau pathology and $60 \%$ of FK506-treated animals survived to one year compared to only $20 \%$ of untreated mice [18]. Some of the treated mice had very little tau pathology and no overt hippocampal atrophy even several months after the development of tau tangles in untreated mice. This suggests that abolishing the inflammation caused by the accumulation of tau might be a new therapy for neurodegenerative disorders like $\mathrm{AD}$ and related tauopathies. It also 
suggests that FK506, or related ligands that bind FK-binding protein (FKBP) immunophilins, may have therapeutic benefit for FTD tauopathy patients. This could occur via FK506-FKBP complexes that inhibit protein phosphatases $2 \mathrm{~B}$, so that nuclear factor of activated T-cells (NFAT) remains phosphorylated, does not enter nuclei, and thereby leads to suppression of immune function, or it could occur by immunophilin-mediated chaperone or peptidylprolyl cis-trans isomerase activity, or other unknown mechanisms. While much more work needs to be done on mechanisms of action of FK506, it is an FDA approved drug used for the treatment of organ rejection, so there is a large existing data set on FK506 that could accelerate efforts to launch a clinical trial of this drug in FTD patients.

\section{Conclusions}

Based on the foregoing and further discussions with the entire Workshop on FTD participants, the FTD Therapy Working Group made the following recommendations for advancing efforts to develop better therapies for FTDs.

\section{Summary Of Consensus FTD Therapy Recommendations \\ Patient Oriented Translational Opportunities}

1. Implement FTD drug trials of $\mathrm{Li}$, Valproate, HSP90 inhibitors (from NCI) in partnership with NINDS, NIA (ADCS), NCI, AFTD, others

2. Prevention trials in hereditary FTDs with diet, physical and cognitive exercise, minimizing metabolic syndrome, etc. in collaboration with Center for Disease Control and the Alzheimer Association

\section{Pre-clinical Translational Opportunities}

1. Proof of concept of Tg mouse studies of GSK/CDK5 inhibitors, chaperones, MT stabilizers, anti-fibrillization/aggregation, Modulation of tau/TDP43/progranulin levels with gene or siRNA therapies, neuroprotection (e.g. FK506), increase clearance of tau and TDP43 aggregates

2. Accelerate drug discovery/high throughput screening (HTS) of compound libraries

3. Make natural product libraries available to FTD scientists

4. Implement partnerships with NIA, NINDS, NCI, and the NIH Molecular Libraries Program as well as the Institute for the Study of Aging (also known as the Alzheimer's Drug Discovery Foundation), the AFTD, and the Alzheimer Association

5. Workshop on best practices for animal model poof of concept studies

\section{Basic Science Opportunities}

1. Increase efforts to understand basic mechanisms of tau, TDP43, PRGN, VCP, etc. mediated neurodegeneration.

2. Increase efforts to develop models of tau, TDP-43, PRGN, VCP, etc. mediated neurodegeneration to model FTDs in worms, flies, mice

\section{References}

1. Ahn JS, Radhakrishnan ML, Mapelli M, Choi S, Tidor B, Cuny GD, Musacchio A, Yeh LA, Kosik KS. Defining Cdk5 ligand chemical space with small molecule inhibitors of tau phosphorylation. Chemical Biology 2005;12:811-823.

2. Bhat RV, Budd Haeberlein SL, Avila J. Glycogen synthase kinase 3: a drug target for CNS therapies. J Neurochem 2004;89:1313-1317. [PubMed: 15189333] 
3. Cairns NJ, Bigio EH, Mackenzie IRA, Neumann M, Lee VM-Y, Hatanpaa KJ, White CL III, Schneider JA, Grinberg LT, Halliday G, Duyckaerts C, Lowe JS, Holm IE, Tolnay M, Okamoto K, Yokoo H, Murayama S, Woulfe J, Dickson DW, Trojanowski JQ, Mann DMA. Neuropathologic diagnostic and nosologic criteria for frontotemporal lobar degeneration: Consensus of the Consortium for Frontotemporal Lobar Degeneration. Acta Neuropath. 2007In press

4. Churcher I. Tau therapeutic strategies for the treatment of Alzheimer's disease. Curr Top Med Chem 2006;6:579-595. [PubMed: 16712493]

5. Dickey CA, Dunmore J, Lu B, Wang J, Lee WC, Kamal A, Burrows F, Eckman C, Hutton M, Petrucelli L. HSP induction mediates selective clearance of tau phosphyorylated at praline-directed Ser/Thr sites but not KXGS (MARK) sites. FASEB J 2006;6:753-755. [PubMed: 16464956]

6. Dickey CA, Eriksen J, Kamal A, Burrows F, Kasibhatla S, Eckman CB, Hutton M, Petrucelli L. Development of a high throughput drug screening assay for the detection of changes in tau levels: Proof of concept with HSP90 inhibitors. Curr Alzheimer Res 2005;2:231-239. [PubMed: 15974923]

7. Engel T, Goni-Oliver P, Lucas JJ, Avila J, Hernandez F. Chronic lithium administration to FTDP-17 tau and GSK-3beta overexpressing mice prevents tau hyperphosphorylation and neurofibrillary tangle formation, but pre-formed neurofibrillary tangles do not revert. J Neurochem 2006;99:1445-1455. [PubMed: 17059563]

8. Fillit HM, Refolo LM. Advancing drug discovery for Alzheimer's disease. Curr Alzheimer Res 2005;2:105-109. [PubMed: 15974904]

9. Kuret J, Congdon EE, Li G, Yin H, Yu X, Zhong Q. Evaluating triggers and enhancers of tau fibrillizatoin. Microscopy Research and Technique 2005;67:141-155. [PubMed: 16103995]

10. Kwong, K.; Neuman, M.; Samapathu, D.; Lee, VM-Y.; Trojanowski, JQ. Acta Neuropath. 2007. TDP-43 proteinopathy: The neuropathology underlying major forms of sporadic and hereditary frontotemporal lobar degeneration and motor neuron disease. In press

11. Le Corre S, Klafki HW, Plesnila N, Hubinger G, Obermeier A, Sahagun H, Monse B, Seneci P, Lewis J, Eriksen J, Zehr C, Yue M, McGowan E, Dickson DW, Hutton M, Roder HM. An inhibitor of tau hyperphosphorylation prevents severe motor impairments in tau transgenic mice. Proc Natl Acad Sci USA 2006;103:9673-9678. [PubMed: 16769887]

12. Lee VM-Y, Trojanowski JQ. Progress from Alzheimer's tangles to pathological tau points towards more effective therapies now. J Alzheimer's Disease Supplement. Alzheimer's Disease: A Century Of Scientific and Clinical Research 2006;9:257-262.

13. Neumann M, Sampathu DM, Kwong LK, Traux A, Miscenyi M, Chou TT, Bruce J, Schuck T, Grossman M, Clark C, McKluskey L, Miller BL, Masliah E, Mackenzie IR, Feldman H, Feiden W, Kretzschmar HA, Trojanowski JQ, Lee VM-Y. Ubiquitinated TDP-43 in frontotemporal lobar degeneration and amyotrophic lateral sclerosis. Science 2006;314:130-133. [PubMed: 17023659]

14. Noble W, Planel E, Zehr C, Olm V, Meyerson J, Suleman F, Gaynor K, Wang L, Lafrancois J, Feinstein B, Burns M, Krishnamurthy P, Wen Y, Bhat R, Lewis J, Dickson D, Duff K. Inhibition of glycogen synthase kinase- 3 by lithium correlates with reduced tauopathy and degeneration in vivo. Proc Natl Acad Sci USA 2005;102:6990-6995. [PubMed: 15867159]

15. Pickhardt M, Gazova Z, von Bergen M, Khlistunova I, Wang Y, Hascher A, Mandelkow EM, Biernat J, Mandelkow E. Anthraquinones inhibit tau aggregation and dissolve Alzheimer's paired helical filaments in vitro and in cells. J Biol Chem 2005;280:3628-2635. [PubMed: 15525637]

16. Roder HM, Hutton ML. Microtubule-associated protein tau as a therapeutic target in neurodegenerative disease. Expert Opin Ther Targets 2007;11:435-442. [PubMed: 17373874]

17. Skovronsky DM, Lee VM-Y, Trojanowski JQ. Neurodegenerative diseases: New concepts of pathogenesis and their therapeutic implications. Annu Rev Pathol Mech Dis 2006;1:151-170.

18. Yoshiyama Y, Higuchi M, Zhang B, Huang S-M, Iwata N, Saido TC, Maeda J, Suhara T, Trojanowski JQ, Lee VM-Y. Synapse loss and microglial activation precede tangles in a P301S tauopathy mouse model. Neuron 2007;53:337-351. [PubMed: 17270732]

19. Zhang B, Maiti A, Shively S, Lakhani F, McDonald-Jones G, Bruce J, Lee EB, Xie SX, Joyce S, Li C, Toleikis PM, Lee VM-Y, Trojanowski JQ. Microtubule binding drugs offset tau sequestration by stabilizing microtubules and reversing fast axonal transport deficits in a murine neurodegenerative tauopathy model. Proc Natl Acad Sci USA 2006;102:227-231. [PubMed: 15615853] 